\title{
An Approach to Preamble Collision Reduction in Grant-Free Random Access with Massive MIMO
}

\author{
Jinho Choi
}

\begin{abstract}
In this paper, we study grant-free random access with massive multiple input multiple output (MIMO) systems. We first show that the performance of massive MIMO based grantfree random access is mainly decided by the probability of preamble collision. The implication of this is that although the number of antennas can be arbitrarily large, the (average) number of successful packet transmissions can be limited by the number of preambles. Then, we propose an approach to preamble collision reduction without increasing the number of preambles using the notion of superpositioned preambles (S-preambles). Since the channel estimation for conjugate beamforming can be carried out when unused $S$-preambles are known, a simple approach is derived to detect unused S-preambles and its performance is analyzed for a special case (superpositions with 2 preambles). Based on analysis and simulation results, it is confirmed that the proposed approach with $S$-preambles can increase the success probability with the same spectral efficiency as the conventional approach.
\end{abstract}

Index Terms-Machine-Type Communication; Preamble Collision; Superposition of Preambles

\section{INTRODUCTION}

Machine-type communication (MTC) has been extensively studied as it plays a crucial role in providing the connectivity for devices in 5th generation (5G) cellular systems and the Internet of Things (IoT) [1] [2]. In MTC, although there are a large number of devices to be connected, in general, they have short data packets with sporadic activity. Thus, random access is employed for MTC thanks to low signaling overhead [3] [4] [5] [6].

To support massive connectivity for a large number of IoT devices and sensors, the notion of massive multiple input multiple output (MIMO) [7] can be considered with a base station (BS) equipped with a large number of antenna elements. Based on [8], it seems that massive MIMO is a solution to massive MTC, as the capacity becomes unbounded in the presence of pilot contamination, which may allow to support a very large number of devices in each cell. In particular, various random access schemes with massive MIMO are studied for massive MTC in [9] [10] [11] [12], where grant-free random access is considered to take advantage of high spatial resolution or selectivity in massive MIMO. Note that grant-free random access is also called two-step random access or one-shot approaches [13] [14] [15].

In [9] [11] [12], the random access schemes with massive MIMO have two phases for grant-free random access. In the first phase, an active device sends a preamble that is

The author is with the School of Information Technology, Deakin University, Geelong, VIC 3220, Australia (e-mail: jinho.choi@deakin.edu.au). This research was supported by the Australian Government through the Australian Research Council's Discovery Projects funding scheme (DP200100391). randomly chosen from a predetermined preamble pool (i.e., a finite set of sequences) so that the BS is able to estimate the channel state information (CSI) of the device. In the second phase, the active device transmits its data packet and the BS performs beamforming with estimated CSI to decode data packets from multiple active devices. The resulting scheme is grant-free random access as no handshaking for request-grant is employed (i.e., the BS does not send any feedback signal to active devices for granting access or reserving channels). Provided that each active device's channel is different from others (due to high spatial resolution or selectivity in massive MIMO), the BS is able to form multiple beams to decode data packets from multiple active devices, which makes grantfree random access with massive MIMO suitable for massive connectivity.

In grant-free random access with massive MIMO, there are various issues including device activity detection [11] and preamble design [16]. Among those, as discussed in [16], preamble design plays a key role in providing a good performance in terms of the probability of successful decoding or success probability. In this paper, since the success probability mainly depends on the probability of preamble collision, without increasing the size of preamble pool, we propose an approach to preamble collision reduction, which results in the decrease of the probability of preamble collision or the increase of success probability. In particular, using superposition of a limited number of orthogonal sequences, a large number of preambles, which are referred to as superpositioned preambles (S-preambles), are generated to lower the the probability of preamble collision. Furthermore, thanks to the fact that each S-preamble is a sum of orthogonal sequences, it is possible to estimate the channel vectors without interference from other preambles, which leads to a better performance than that of conventional non-orthogonal preambles (e.g., Zadoff-Chu (ZC) or Gaussian random sequences) [17]. With a special case of superpositioned preambles (i.e., superpositions with two preambles), we derive a low-complexity preamble detection method and analyze the performance in the paper.

Note that in [16], transmitting multiple consecutive preambles is considered to increase the success probability, which unfortunately leads to the increase of the length of preamble transmission phase and a poor spectral efficiency. On the other hand, in the proposed approach, the length of the preamble transmission phase is the same as that of preamble (as in conventional approaches, e.g., [12]). In other words, with the same spectral efficiency as the conventional grant-free random access with massive MIMO, a higher success probability can be achieved.

It is also possible to decrease the probability of preamble 
collision by increasing the number of preambles. With a fixed length of preambles (or a fixed system bandwidth), it is possible to have a large number of preambles if preambles are not orthogonal. In this case, the preamble detection can be seen as a sparse signal recovery problem in the context of compressive sensing (CS) [18] [19], and various CS algorithms can be applied to the preamble detection (or user activity detection) as in [10] [11] [20] [21]. In general, the complexity of CS algorithms is high (usually proportional to the number of preambles) for joint activity detection. On the other hand, in this paper, since a set of orthogonal sequences is used to increase the number of preambles, simple low-complexity correlators can be used for the preamble detection, which means that the complexity is low as it is dependent on the length of preambles. Consequently, S-preambles allow us not only to use the low-complexity channel estimation (as the case of orthogonal preambles), but also to lower the probability of collision (as the case of non-orthogonal preambles), which results in a better performance.

The rest of the paper is organized as follows. In Section II, we present the system model for grant-free random access with massive MIMO, and show that the performance is mainly limited by the size of preamble pool. Then, we propose an approach to preamble collision reduction without increasing the size of preamble pool (or the length of preamble transmission phase) in Section III and derive a hypothesis testing approach to detect unused preambles that is necessary for the channel estimation in Section IV. In Section V, we present simulation results. The paper is concluded in Section VI with some remarks.

Notation: Matrices and vectors are denoted by upper- and lower-case boldface letters, respectively. The superscripts $\mathrm{T}$ and $\mathrm{H}$ denote the transpose and complex conjugate, respectively. The Kronecker product is denoted by $\otimes . \mathbb{E}[\cdot]$ and $\operatorname{Var}(\cdot)$ denote the statistical expectation and variance, respectively. $\mathcal{C N}(\mathbf{a}, \mathbf{R})$ represents the distribution of circularly symmetric complex Gaussian (CSCG) random vectors with mean vector a and covariance matrix $\mathbf{R}$. The $\mathrm{Q}$-function is given by $\mathcal{Q}(x)=\int_{x}^{\infty} \frac{1}{\sqrt{2 \pi}} e^{-\frac{t^{2}}{2}} d t$.

\section{SySTEM MODEL}

Suppose that a grant-free random access system consists of a BS and multiple devices. It is assumed that the BS is equipped with $M$ antenna elements and each device has a single antenna.

\section{A. Grant-free Random Access}

In grant-free or 2-step random access, there are two phases [12] [16]. The first phase is the preamble transmission phase and the following phase is the data transmission phase as illustrated in Fig. 1. In the preamble transmission phase, each active device with data is to send a randomly selected preamble from the following preamble pool: $\mathcal{C}=\left\{\mathbf{c}_{1}, \ldots, \mathbf{c}_{L}\right\}$, where $\mathbf{c}_{l} \in \mathbb{C}^{N \times 1}$ represents the $l$ th preamble. Throughout the paper, it is assumed that the $\mathbf{c}_{l}$ 's are orthonormal sequences of length $N$ and $\left|\left[\mathbf{c}_{l}\right]_{n}\right|=\frac{1}{\sqrt{N}}$ for all $l$ and $n$. Thus, we assume that $L=N$. After sending a preamble, an active device sends its data packet in the data transmission phase as shown in Fig. 1. Throughout the paper, it is assumed that the lengths of data packets of devices are the same. In addition, all the devices are synchronized (to this end, the BS needs periodically broadcast a beacon signal).

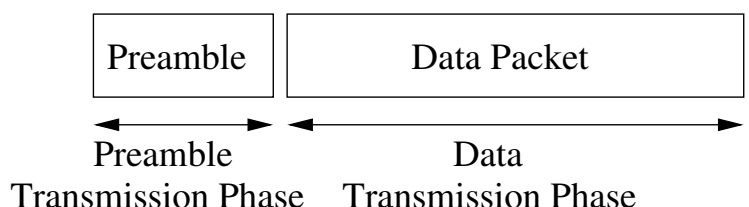

Fig. 1. Two phases (i.e., preamble transmission and data transmission phases) for a grant-free random access scheme.

Let $\mathbf{h}_{k} \in \mathbb{C}^{M \times 1}$ represent the channel vector from active device $k$ to the $\mathrm{BS}$, which remains unchanged for the transmission slot consisting of consecutive preamble and data transmission phases. Then, in the preamble transmission phase, the BS receives the following signal in the space and time domain:

$$
\mathbf{Y}=\sum_{k=1}^{K} \mathbf{h}_{k} \sqrt{P_{k}} \mathbf{c}_{l(k)}^{\mathrm{T}}+\mathbf{N} \in \mathbb{C}^{M \times N}
$$

where $K$ represents the number of active devices, $l(k)$ denotes the index of the preamble chosen by active device $k, P_{k}$ is the transmit power of active device $k$, and $[\mathbf{N}]_{m, n} \sim \mathcal{C N}\left(0, N_{0}\right)$ is the background noise at the $m$ th antenna and the $n$th preamble symbol duration.

Each active device transmits its data packet of length $D$ during the data transmission phase. The corresponding received signal at the BS becomes

$$
\mathbf{Z}=\sum_{k=1}^{K} \mathbf{h}_{k} \sqrt{P_{k}} \mathbf{b}_{k}^{\mathrm{T}}+\tilde{\mathbf{N}} \in \mathbb{C}^{M \times D},
$$

where $\mathbf{b}_{k}$ represents the data packet from active device $k$ and $[\tilde{\mathbf{N}}]_{m, d} \sim \mathcal{C} \mathcal{N}\left(0, N_{0}\right)$ is the background noise at the $m$ th antenna and the $n$th data symbol duration. Throughout the paper, we assume that $\mathbb{E}\left[\mathbf{b}_{k}\right]=\mathbf{0}$ and $\mathbb{E}\left[\mathbf{b}_{k} \mathbf{b}_{k}^{\mathrm{H}}\right]=\mathbf{I}$ (i.e., the symbol energy is normalized).

The BS uses a bank of $L$ correlators to detect transmitted preambles and estimate the channel vectors of the devices that associate with the detected preambles. The output of the correlator with $\mathbf{c}_{l}$ is given by

$$
\begin{aligned}
\mathbf{g}_{l} & =\mathbf{Y} \mathbf{c}_{l}=\sum_{k=1}^{K} \mathbf{h}_{k} \sqrt{P_{k}} \delta_{l(k), l}+\mathbf{N} \mathbf{c}_{l} \\
& =\sum_{k \in \mathcal{K}_{l}} \mathbf{h}_{k} \sqrt{P_{k}}+\mathbf{N} \mathbf{c}_{l},
\end{aligned}
$$

where $\delta_{l, l^{\prime}}$ is the Kronecker delta (i.e., $\delta_{l, l^{\prime}}=1$ if $l=l^{\prime}$, and 0 otherwise) and $\mathcal{K}_{l}$ represents the index set of the active devices that choose preamble $l$. If active device $k$ is the only device that chooses preamble $l$ (i.e., $l(k)=l$ and $\mathcal{K}_{l}=\{k\}$ ), thanks to the orthogonality of preambles, it can be shown that

$$
\mathbf{g}_{l}=\mathbf{h}_{k} \sqrt{P_{k}}+\mathbf{n}_{l}
$$


where $\mathbf{n}_{l}=\mathbf{N c}_{l} \sim \mathcal{C N}\left(\mathbf{0}, N_{0} \mathbf{I}\right)$. To decode the data packet from active device $k$, conjugate beamforming is applied and the output of the beamformer becomes

$$
\begin{aligned}
\mathbf{x}_{l} & =\mathbf{g}_{l}^{\mathrm{H}} \mathbf{Z} \\
& =\mathbf{g}_{l}^{\mathrm{H}} \mathbf{h}_{k} \sqrt{P_{k}} \mathbf{b}_{k}+\sum_{k^{\prime} \neq k} \mathbf{g}_{l}^{\mathrm{H}} \mathbf{h}_{k^{\prime}} \sqrt{P_{k^{\prime}}} \mathbf{b}_{k^{\prime}}^{\mathrm{T}}+\mathbf{g}_{l}^{\mathrm{H}} \tilde{\mathbf{N}} .
\end{aligned}
$$

If $\left|\mathbf{g}_{l}^{\mathrm{H}} \mathbf{h}_{k}\right|^{2}$ is sufficiently larger than $\left|\mathbf{g}_{l}^{\mathrm{H}} \mathbf{h}_{k^{\prime}}\right|^{2}$, a high signalto-interference-plus-noise ratio (SINR) can be achieved for successful decoding.

Throughout this paper, as in [7], let $\mathbf{h}_{k}=\sqrt{\ell_{k}} \mathbf{v}_{k}$, where $\ell_{k}$ represents the large-scale fading coefficient that depends on the distance between the $k$ th active device and the BS and $\mathbf{v}_{k}$ stands for the small-scale fading vector. For tractable analysis, we consider the following assumption [22].

A) Throughout the paper, $P_{k}$ is decided to be inversely proportional to $\ell_{k}$ via power control so that

$$
\mathbf{h}_{k} \sqrt{P_{k}}=\mathbf{v}_{k} \sqrt{P_{\mathrm{rx}}}
$$

where $P_{\mathrm{rx}}$ represents the (average) receive signal power and $\mathbf{v}_{k} \sim \mathcal{C N}(\mathbf{0}, \mathbf{I})$ is independent for all $k$ (i.e., Rayleigh fading is assumed for small-scale fading).

In addition, for convenience, let $\gamma=\frac{P_{\mathrm{rx}}}{N_{0}}$ be the signal-to-noise ratio (SNR).

\section{B. Size of Preamble Pool and Preamble Collision}

Suppose that all $K$ active devices randomly choose preambles from $\mathcal{C}$. The probability that one active device is not collided with the other active devices or one active device is collision-free is given by $\mathbb{P}_{\mathrm{cf}}=\left(1-\frac{1}{L}\right)^{K-1} \leq e^{-\frac{K-1}{L}}$. For active device $k$, the probability that its signal is successfully decoded (i.e., the SINR is higher than a decoding threshold without preamble collision) is given by

$$
\eta_{k}=\mathbb{P}_{\mathrm{cf}} \operatorname{Pr}\left(\mathrm{SINR}_{k} \geq \Omega\right)
$$

where $\Omega>0$ is the SINR threshold for successful decoding. For convenience, in short, $\eta_{k}$ is referred to as the success probability. In [12], closed-form expressions for $\operatorname{Pr}\left(\operatorname{SINR}_{k} \geq \Omega\right)$ are derived with various beamforming approaches. In general, if $M$ is sufficiently large, for a finite $\Omega, \operatorname{Pr}\left(\operatorname{SINR}_{k} \geq \Omega\right)$ approaches 1 (as the SINR grows linearly with $M$ on average). Thus, we can see that the performance limiting factor (for each active device) is $\mathbb{P}_{c f}$ that depends on the size of preamble pool, $L$. Thus, for orthogonal preambles, to increase $L$, the length of preambles needs to increase, which unfortunately results in a poor spectral efficiency (e.g., as in [16]).

\section{An EfFective Approach to Preamble Collision REDUCTION}

In this section, we propose an approach to preamble collision reduction without increasing the size of preamble pool, $L$, which can effectively improve the success probability of each active device.

\section{A. Transmission of Multiple Preambles per Device}

While each active device is to choose only one preamble to transmit in the preamble transmission phase in the conventional approach, we propose to choose $B(\geq 1)$ multiple preambles by each active device. An active device is to send a superposition of $B$ different preambles, which is called a S-preamble. Then, the number of possible selections becomes $Q=\left(\begin{array}{l}L \\ B\end{array}\right)$. Let $\mathcal{A}_{q}$ denote the index set of the $q$ th selection, $q=1, \ldots, Q$. For example, with $L=4$ and $B=2$, we can have

$$
\begin{gathered}
\mathcal{A}_{1}=\{1,2\}, \mathcal{A}_{2}=\{1,3\}, \mathcal{A}_{3}=\{1,4\}, \mathcal{A}_{4}=\{2,3\}, \\
\mathcal{A}_{5}=\{2,4\}, \text { and } \mathcal{A}_{6}=\{3,4\} .
\end{gathered}
$$

Then, the $q$ th $\mathrm{S}$-preamble can be defined as

$$
\mathbf{s}_{q}=\sum_{l \in \mathcal{A}_{q}} \mathbf{c}_{l} .
$$

In the proposed approach, the received signal in the preamble transmission phase is given by

$$
\mathbf{Y}=\sum_{k=1}^{K} \mathbf{h}_{k} \mathbf{s}_{q(k)}^{\mathrm{T}}+\mathbf{N} \in \mathbb{C}^{M \times N}
$$

where $q(k)$ denotes the S-preamble index chosen by active device $k$. For convenience, let $\mathcal{Q}$ be the index set of the $\mathrm{S}$ preambles chosen by $K$ active devices.

In summary, the proposed approach is a grant-free or 2step approach where an active device transmits a sum of $B$ orthogonal preambles in the preamble transmission phase. Thus, it has the following steps:

$\mathrm{S} 1)$ In the first step, an active device chooses one of $Q \mathrm{~S}$ preambles uniformly at random and transmits it, which is a sum of $B$ orthogonal preambles, followed by a data packet transmitted in the data transmission phase as shown in Fig. 1.

S2) In the second step, the BS is to detect transmitted preambles and estimate the channel vectors of the active devices associated with transmitted preambles. In Subsection III-B, we will discuss a low-complexity channel estimation method by taking advantage of S-preambles. With the estimated channel vectors, the BS decodes the data packets and sends feedback signals (of decoding outcomes) to active devices.

It is clear that the probability that one active device is not collided with the other active devices in terms of S-preambles is given by

$$
\mathbb{P}_{\mathrm{cf}}=\left(1-\frac{1}{Q}\right)^{K-1} .
$$

For example, suppose that $L=64$ and $K=10$. Then, if $B=$ 1 (i.e., the conventional approach), the probability that one active device is collision-free is $\left(1-\frac{1}{L}\right)^{K-1} \approx 0.867$. When $\mathrm{S}$-preambles are used with $B=2$ in the proposed approach, this probability becomes $\left(1-\frac{1}{Q}\right)^{K-1} \approx 0.995$.

In Fig. 2, we show the probability that one active device is not collided with the other active devices for different numbers 
of active devices when $L=64$ and $B=2$. Clearly, using Spreambles, the probability becomes significantly high, which results in the increase of the success probability as shown in (7). Note that in (7), $\operatorname{Pr}\left(\operatorname{SINR}_{k} \geq \Omega\right)$ is not related to the type of preambles.

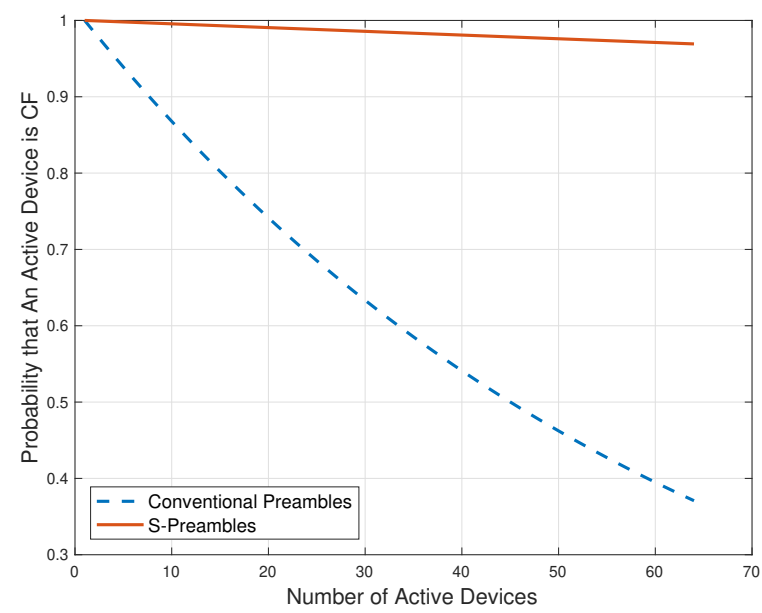

Fig. 2. Probability that one active device is collision-free in terms of Spreambles for different numbers of active devices when $L=64$ and $B=2$.

Since $Q$ increases with $B\left(\leq\left\lfloor\frac{L}{2}\right\rfloor\right)$, we can see that the probability decreases with $B$. Therefore, it is possible to reduce preamble collisions and improve the performance.

\section{B. Channel Estimation with Known Transmitted S-Preambles}

In the rest of the paper, for simplicity, we mainly focus on the case that $B=2$ and discuss the channel estimation with known transmitted S-preambles.

For convenience, suppose that each $\mathrm{S}$-preamble is associated with one device (without any collision). In addition, the device associated with the $q$ th S-preamble is referred to as the $q$ th virtual active device. The associated virtual channel vector is denoted by $\mathbf{a}_{q}$. If the $q$ th S-preamble is transmitted, the $q$ th virtual active device is one of active devices. On the other hand, if the $q$ th S-preamble is not transmitted, the $q$ th virtual active device is not an active device. With $Q$ virtual active devices, the received signal in the preamble transmission phase can be rewritten as

$$
\mathbf{Y}=\sum_{q=1}^{Q} \sqrt{P_{\mathrm{rx}}} \mathbf{a}_{q} \mathbf{s}_{q}^{\mathrm{T}}+\mathbf{N}
$$

In order to show how S-preambles work together with virtual channel vectors, it might be useful to consider a simple example with $L=4$. Then, as mentioned earlier, there are $Q=\left(\begin{array}{l}4 \\ 2\end{array}\right)=6$ possible choices as in (8). In this case, without background noise and receive power term, from (12), we have

$$
\mathbf{g}_{l}=\mathbf{Y} \mathbf{c}_{l}=\left(\sum_{q} \mathbf{a}_{q}\left(\sum_{l^{\prime} \in \mathcal{A}_{q}} \mathbf{c}_{l^{\prime}}\right)^{\mathrm{T}}\right) \mathbf{c}_{l} .
$$

For example, we have $\mathbf{g}_{1}=\mathbf{a}_{1}+\mathbf{a}_{2}+\mathbf{a}_{3}, \mathbf{g}_{2}=\mathbf{a}_{1}+\mathbf{a}_{4}+\mathbf{a}_{5}$, and so on. Thus, it can be shown that

$$
\mathbf{g}=\left[\begin{array}{l}
\mathbf{g}_{1} \\
\mathbf{g}_{2} \\
\mathbf{g}_{3} \\
\mathbf{g}_{4}
\end{array}\right]=(\mathbf{\Phi} \otimes \mathbf{I})\left[\begin{array}{llllll}
\mathbf{a}_{1}^{\mathrm{T}} & \mathbf{a}_{2}^{\mathrm{T}} & \mathbf{a}_{3}^{\mathrm{T}} & \mathbf{a}_{4}^{\mathrm{T}} & \mathbf{a}_{5}^{\mathrm{T}} & \mathbf{a}_{6}^{\mathrm{T}}
\end{array}\right]^{\mathrm{T}}
$$

where

$$
\boldsymbol{\Phi}=\left[\begin{array}{llllll}
1 & 1 & 1 & 0 & 0 & 0 \\
1 & 0 & 0 & 1 & 1 & 0 \\
0 & 1 & 0 & 1 & 0 & 1 \\
0 & 0 & 1 & 0 & 1 & 1
\end{array}\right]
$$

In general, the size of $\boldsymbol{\Phi}$ is $L \times Q$. If a bipartite graph with $L$ left vertices and $Q$ right vertices is considered, $\Phi$ can be seen as a transfer or biadjacency matrix [23]. Furthermore, it can be shown that the number of 1's of each column is 2 and that of each row is $L-1$. Thus, $\boldsymbol{\Phi}$ is the transfer matrix of a bipartite graph with $L$ left vertices and $Q$ right vertices that is $(L-1)$-regular on the left and 2-regular on the right.

Note that if $B$ is greater than $2, \boldsymbol{\Phi}$ becomes the transfer matrix of a bipartite graph with $L$ left vertices and $Q=\left(\begin{array}{l}L \\ B\end{array}\right)$ right vertices that is $\left(\begin{array}{l}L-1 \\ B-1\end{array}\right)$-regular on the left and $B$-regular on the right. Thus, for $B \geq 3$, the resulting bipartite graph becomes an expander graph. Since we only focus on the case of $B=2$ in this paper, a generalization with $B \geq 3$ is to be studied in the future as a further research topic.

In (12), since the $\mathbf{a}_{q}$ 's are virtual, if no active device chooses the $q$ th $\mathrm{S}$-preamble, we have $\mathbf{a}_{q}=\mathbf{0}$ and it is not necessary to estimate the corresponding channel. In other words, we only need to estimate the channels associated with the transmitted (or chosen) S-preambles. For convenience, let $\bar{K}$ be the number of the virtual channels associated with the transmitted S-preambles. If there is no preamble collision, $\bar{K}$ becomes $K$. Suppose that $\bar{K} \leq L$ and denote by $\overline{\boldsymbol{\Phi}}$ the submatrix of $\boldsymbol{\Phi}$ obtained by taking the columns corresponding to the transmitted S-preambles. Then, for example, using the least squares (LS) approach [24], the $\bar{K}$ channel vectors can be estimated as follows:

$$
\hat{\mathbf{a}}=\left(\overline{\boldsymbol{\Phi}}^{\dagger} \otimes \mathbf{I}\right) \mathbf{g},
$$

where $\overline{\boldsymbol{\Phi}}^{\dagger}$ represents the Moore-Penrose pseudoinverse of $\overline{\boldsymbol{\Phi}}$ and $\hat{\mathbf{a}}$ is a vector of the estimates of $\bar{K}$ virtual channel vectors.

Clearly, in order to estimate the channel vectors as above, it is important to detect unused S-preambles. We discuss an approach based on the outputs of correlators as follows.

With $B=2$, recall that $\mathcal{A}_{q}=\left\{q_{1}, q_{2}\right\}$ is the set of the two indices of the preambles used in the $q$ th S-preamble, i.e., $q_{1}$ and $q_{2}$, where $q_{b} \in\{1, \ldots, L\}$. For the $q$ th $\mathrm{S}$-preamble, the BS can use the following correlators' outputs:

$$
\mathbf{g}_{q, 1}=\mathbf{Y} \mathbf{c}_{q_{1}} \text { and } \mathbf{g}_{q, 2}=\mathbf{Y} \mathbf{c}_{q_{2}} .
$$

Example 1: With $L=4$, suppose that there are 3 active devices, i.e., $K=3$, that choose $\mathrm{S}$-preambles 1 , 3 , and 4 , 
i.e., $\mathcal{Q}=\{1,3,4\}$. In this case, without background noise and receive power term, it can be shown that

$$
\begin{aligned}
& q=1:\left\{\mathbf{g}_{1,1}, \mathbf{g}_{1,2}\right\}=\left\{\mathbf{v}_{1}+\mathbf{v}_{2}, \mathbf{v}_{1}+\mathbf{v}_{3}\right\} \\
& q=2:\left\{\mathbf{g}_{2,1}, \mathbf{g}_{2,2}\right\}=\left\{\mathbf{v}_{1}+\mathbf{v}_{2}, \mathbf{v}_{3}\right\} \\
& q=3:\left\{\mathbf{g}_{3,1}, \mathbf{g}_{3,2}\right\}=\left\{\mathbf{v}_{1}+\mathbf{v}_{2}, \mathbf{v}_{2}\right\} \\
& q=4:\left\{\mathbf{g}_{4,1}, \mathbf{g}_{4,2}\right\}=\left\{\mathbf{v}_{1}+\mathbf{v}_{3}, \mathbf{v}_{3}\right\} \\
& q=5: \quad\left\{\mathbf{g}_{5,1}, \mathbf{g}_{5,2}\right\}=\left\{\mathbf{v}_{1}+\mathbf{v}_{3}, \mathbf{v}_{2}\right\} \\
& q=6:\left\{\mathbf{g}_{6,1}, \mathbf{g}_{6,2}\right\}=\left\{\mathbf{v}_{3}, \mathbf{v}_{2}\right\} .
\end{aligned}
$$

Since active device 1 chooses the first S-preamble, in both $\mathbf{g}_{1,1}$ and $\mathbf{g}_{1,2}, \mathbf{v}_{1}$ can be found. For the pairs of $\left\{\mathbf{g}_{q, 1}, \mathbf{g}_{q, 2}\right\}$ corresponding to the $\mathrm{S}$-preambles chosen by the other active devices, we have the same observation. On the other hand, the pairs of $\left\{\mathbf{g}_{q, 1}, \mathbf{g}_{q, 2}\right\}$ corresponding to the S-preambles not chosen by any active devices, there are no common channel vectors in both $\mathbf{g}_{q, 1}$ and $\mathbf{g}_{q, 2}$. Based on the above observation, we have the following result.

Lemma 1: From (10) and (15), it can be shown that

$$
\begin{aligned}
\mathbf{g}_{q, 1} & =\sum_{k=1}^{K} \mathbf{v}_{k} \sqrt{P_{\mathrm{rx}}} \delta_{\mathcal{A}_{q(k)}(1), q_{1}}+\mathbf{N} \mathbf{c}_{q_{1}} \\
\mathbf{g}_{q, 2} & =\sum_{k=1}^{K} \mathbf{v}_{k} \sqrt{P_{\mathrm{rx}}} \delta_{\mathcal{A}_{q(k)}(2), q_{2}}+\mathbf{N} \mathbf{c}_{q_{2}},
\end{aligned}
$$

where $\mathcal{A}_{q}(b)$ represents the $b$ th element of $\mathcal{A}_{q}$. Then, if the $q$ th $\mathrm{S}$-preamble is chosen by $K_{q}$ active devices, under the assumption of $\mathbf{A}$ ), w.p. 1, we have

$$
\lim _{M \rightarrow \infty} \frac{\mathbf{g}_{q, 1}^{\mathrm{H}} \mathbf{g}_{q, 2}}{M} \rightarrow P_{\mathrm{rx}} K_{q}
$$

Proof: For a given S-preamble, say S-preamble $q$, since $q_{1}$ and $q_{2}$ are different, based on the strong law of large numbers, w.p. 1 , it follows

$$
\frac{\left(\mathbf{N} \mathbf{c}_{q_{1}}\right)^{\mathrm{H}} \mathbf{N} \mathbf{c}_{q_{2}}}{M} \rightarrow 0,
$$

as $M \rightarrow \infty$. Similarly, under the assumption of $\mathbf{A}$ ),

$$
\frac{\left(\sum_{k=1}^{K} \mathbf{v}_{k} \sqrt{P_{\mathrm{rx}}} \delta_{\mathcal{A}_{q(k)}(b), q_{b}}\right)^{\mathrm{H}} \mathbf{N} \mathbf{c}_{q_{b^{\prime}}}}{M} \rightarrow 0,
$$

as $M \rightarrow \infty$, for $b \neq b^{\prime}$. Since there are $K_{q}$ common channel vectors in both $\sum_{k=1}^{K} \mathbf{v}_{k} \sqrt{P_{\mathrm{rx}}} \delta_{\mathcal{A}_{q(k)}(b), q_{b}}, b=1,2$, we can show that (17) holds.

Consequently, from (17), the correlation between $\mathbf{g}_{q, 1}$ and $\mathbf{g}_{q, 2}$ can be used to decide whether or not the $q$ th S-preamble is chosen by any active device. If the unused S-preambles are detected, the corresponding virtual channels are assumed to be zero vectors. As in Example 1, we can see that the correlation coefficients between $\mathbf{g}_{q, 1}$ and $\mathbf{g}_{q, 2}$ corresponding to $q \in\{2,5,6\}$ would approach 0 . Thus, there are non-zero virtual channel vectors for $q \in\{1,3,4\}$. Then, we have

$$
\overline{\boldsymbol{\Phi}}=\left[\begin{array}{lll}
1 & 1 & 0 \\
1 & 0 & 1 \\
0 & 0 & 1 \\
0 & 1 & 0
\end{array}\right]
$$

in (14) to estimate non-zero virtual channel vectors, $\hat{\mathbf{a}}$. Without any S-preamble collision, the number of columns of $\overline{\boldsymbol{\Phi}}$ is equivalent to the number of active devices. Thus, it is possible that the channel vectors of the active devices can be estimated when $K \leq L$ without S-preamble collision. However, the condition that $K \leq L$ without S-preamble collision is not a sufficient condition for the channel estimation, because the rank of $\overline{\boldsymbol{\Phi}}$ may not be the same as the number of columns (when $K \leq L$ ). For example, when $\mathcal{Q}=\{1,2,5,6\}$ with $K=4$ and $L=4$. the rank of $\overline{\boldsymbol{\Phi}}$ is 3 , not 4 , which means that the channel estimation for $K=4$ active devices is not possible.

In Fig. 3, the (empirical) average $\operatorname{rank}$ of $\overline{\boldsymbol{\Phi}}, \mathbb{E}[\operatorname{rank}(\overline{\boldsymbol{\Phi}})]$, is shown as a function of $K$ when $L=64$ when $K$ (out of $Q$ ) columns of $\boldsymbol{\Phi}$ are randomly selected for $\overline{\boldsymbol{\Phi}}$. It is shown that the difference between the rank of $\overline{\boldsymbol{\Phi}}$ and $K$ increases as $K$ approaches $L$. Thus, although the use of S-preamble can mitigate the preamble collision, due to rank deficient $\overline{\mathbf{\Phi}}$ for a $K$ close to $L$, some channels cannot be estimated. In Section V, we present simulation results that show the impact of rank deficient $\bar{\Phi}$ on the success probability.

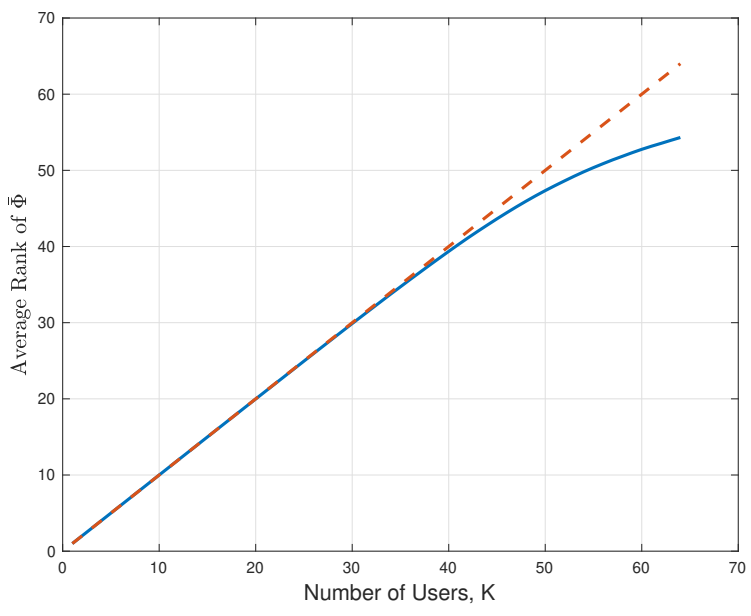

Fig. 3. The average rank of $\overline{\boldsymbol{\Phi}}$ as a function of $K$ when when $K=L=64$, which is shown by the solid line (the dashed line represents the number of the columns of $\overline{\boldsymbol{\Phi}}$, i.e., $K$ ).

There are a few remarks.

- To increase the number of preambles, non-orthogonal preambles can be used. For example, ZC or Gaussian random sequences can be used for preambles [17]. As shown in [17], although the number of preambles can be large (to lower the probability of collision), its performance (in terms of success probability) can be worse than that of orthogonal preambles due to the correlation between preambles that results in degraded channel estimates and SINR.

- It is noteworthy that the channel estimation in (14) is based on the outputs of simple correlators in (3). As a result, the complexity is mainly proportional to the number of multiplications to carry out $\mathbf{Y} \mathbf{c}_{l}$ for all $l$, which is $L^{2} M$. In addition, to find the unused $\mathrm{S}$-preambles, we need to find the correlation between the $\mathbf{g}_{l}$ 's, which requires a complexity order of $O\left(L^{2} M\right)$ with $B=2$. 
Thus, the proposed approach with S-preambles has a low computational complexity (i.e., $O\left(L^{2} M\right)$ ). Note that as mentioned earlier, CS algorithms can be used to detect non-orthogonal preambles (including S-preambles) as in [10] [11] [20] [21]. Since the complexity of CS algorithms depends on the length of preambles as well as the number of preambles, the resulting complexity can be high (e.g., a variational inference algorithm used in [21] requires a complexity of $O\left(Q M L^{2}\right)$ per iteration. Thus, with $B=2$, we have $Q=O\left(L^{2}\right)$, which means that the complexity order becomes $O\left(M L^{4}\right)$ per iteration. Clearly, S-preambles allow us not only to use the lowcomplexity channel estimation studied in this section for grant-free random access with massive MIMO, but also to lower the probability of collision, which results in a better performance.

\section{Hypothesis Testing For the Detection of UNUSED PREAMBLES}

Since Lemma 1 is valid when $M \rightarrow \infty$, with a finite $M$ (but sufficiently large), a hypothesis testing approach [25] is required for the detection of unused S-preambles (or transmitted S-preambles). To this end, let $D=\frac{\mathbf{g}_{q, 1}^{\mathrm{H}} \mathbf{g}_{q, 2}}{\sqrt{M}}$. The distributions of the test statistic $D$ can be found from [26]. For a large $M$, alternatively, we can use the central limit theorem (CLT). For convenience, we only consider the $q$ th S-preamble. In addition, we assume that the number of the active devices choosing preamble $q_{b}, b \in\{1,2\}$, associated with S-preamble $q$ is known, which is denoted by $\kappa_{q_{b}}$. For example, consider Example 1 with $A_{q}=\left\{q_{1}, q_{2}\right\}=\{1,2\}$ when $q=1$. In this case, $\kappa_{q_{1}}=2$ and $\kappa_{q_{2}}=2$.

Let $H_{0}$ represent the hypothesis that S-preamble $q$ is unused and $H_{1}$ the alternative hypothesis. If $\kappa_{q_{b}}=0$ for any $b \in\{1,2\}$, it is clear that S-preamble $q$ is not chosen. Thus, we only focus on the case that $\kappa_{q_{b}}>0$ for $b \in\{1,2\}$. In addition, for $H_{1}$, we only consider the case that $K_{q}=1$ (as the probability that $K_{q} \geq 2$ might be sufficiently low).

Under $H_{0}$, based on the CLT, it is readily shown that $\mathbb{E}[D]=0, \operatorname{Var}(D)=\left(P_{\mathrm{rx}} \kappa_{q_{1}}+N_{0}\right)\left(P_{\mathrm{rx}} \kappa_{q_{2}}+N_{0}\right)$, and $\mathbb{E}\left[D^{2}\right]=0$. For convenience, let $\rho_{b}=P_{\mathrm{rx}} \kappa_{q_{b}}+N_{0}$. Then, under $H_{0}, D \sim \mathcal{C N}\left(0, \rho_{1} \rho_{2}\right)$. The distribution of $D$ under $H_{1}$ can be found as follows.

Lemma 2: Using the CLT, under $H_{1}$, with $K_{q}=1$, it can be shown that

$$
D \sim \mathcal{C N}\left(\sqrt{M} P_{\mathrm{rx}}, \rho_{1} \rho_{2}, P_{\mathrm{rx}}^{2}\right),
$$

where $X \sim \mathcal{C N}\left(\mathbb{E}[X], \operatorname{Var}(X), \mathbb{E}\left[X^{2}\right]-\mathbb{E}[X]^{2}\right)$ represents a complex Gaussian random variable [27]. Note that while $D$ under $H_{0}$ is CSCG, $D$ under $H_{1}$ is not CSCG (as $\mathbb{E}\left[D^{2}\right]-$ $\mathbb{E}[D]^{2} \neq 0$ ).

Proof: With $K_{q}=1$, suppose that the $k$ th active device chooses the $q$ th S-preamble. Then, the $m$ th elements of $\mathbf{g}_{q, b}$, $b \in\{1,2\}$, are given by

$$
\begin{aligned}
& {\left[\mathbf{g}_{q, 1}\right]_{m}=\sqrt{P_{\mathrm{rx}}}\left[\mathbf{v}_{k}\right]_{m}+\nu_{1, m}+n_{1, m}} \\
& {\left[\mathbf{g}_{q, 2}\right]_{m}=\sqrt{P_{\mathrm{rx}}}\left[\mathbf{v}_{k}\right]_{m}+\nu_{2, m}+n_{2, m},}
\end{aligned}
$$

where $n_{b, m}$ represents the $m$ th element of $\mathbf{N c}_{q_{b}}$ and $\nu_{b, m} \sim \mathcal{C N}\left(0, P_{\mathrm{rx}}\left(\kappa_{q_{b}}-1\right)\right)$. As a result, $\nu_{b, m}+n_{b, m} \sim$ $\mathcal{C N}\left(0, P_{\mathrm{rx}}\left(\kappa_{q_{b}}-1\right)+N_{0}\right)$. For convenience, let $I_{b}=P_{\mathrm{rx}}\left(\kappa_{q_{b}}-\right.$ 1) $+N_{0}$.

Since each of $\mathbf{v}_{k} \sim \mathcal{C N}(\mathbf{0}, \mathbf{I})$ is iid, and $n_{1, m}$ and $n_{2, m}$ are independent zero-mean CSCG random variables, we have

$$
\mathbb{E}[D]=\mathbb{E}\left[\frac{1}{\sqrt{M}} \sum_{m}\left(\left[\mathbf{g}_{q, 1}\right]_{m}\right)^{*}\left(\left[\mathbf{g}_{q, 2}\right]_{m}\right)\right]=\sqrt{M} P_{\mathrm{rx}} .
$$

It can also be shown that

$$
\begin{aligned}
\mathbb{E}\left[|D|^{2}\right] & =\frac{1}{M} \mathbb{E}\left[\left|\sum_{m}\left(\left[\mathbf{g}_{q, 1}\right]_{m}\right)^{*}\left(\left[\mathbf{g}_{q, 2}\right]_{m}\right)\right|^{2}\right] \\
& =3 P_{\mathrm{rx}}^{2}+(M-1) P_{\mathrm{rx}}^{2}+\left(I_{1}+I_{2}\right) P_{\mathrm{rx}}+I_{1} I_{2},(21)
\end{aligned}
$$

by using the fact that $\mathbb{E}\left[|X|^{4}\right]=2 \sigma^{4}$ for $X \sim \mathcal{C N}\left(0, \sigma^{2}\right)$. As a result, the variance of $D$ is given by

$$
\operatorname{Var}(D)=P_{\mathrm{rx}}^{2}+\left(I_{1}+I_{2}\right) P_{\mathrm{rx}}+I_{1} I_{2}=\rho_{1} \rho_{2} .
$$

It can also be readily shown that

$$
\mathbb{E}\left[(D-\mathbb{E}[D])^{2}\right]=P_{\mathrm{rx}}^{2},
$$

which completes the proof.

Let $\mathbf{d}=\left[\begin{array}{ll}D & D^{*}\end{array}\right]^{\mathrm{T}}$. In addition, let $\mathbf{R}_{0}=\operatorname{diag}\left(\rho_{1} \rho_{2}, \rho_{1} \rho_{2}\right)$ and

$$
\mathbf{R}_{1}=\left[\begin{array}{cc}
\rho_{1} \rho_{2} & P_{\mathrm{rx}}^{2} \\
P_{\mathrm{rx}}^{2} & \rho_{1} \rho_{2}
\end{array}\right] .
$$

Then, according to [28] [27], it can be shown that

$$
f\left(\mathbf{d} \mid H_{i}\right)=\frac{1}{\pi\left(\operatorname{det} \mathbf{R}_{i}\right)^{-\frac{1}{2}}} e^{-\frac{1}{2}\left(\mathbf{d}-\mathbf{m}_{i}\right)^{\mathrm{H}} \mathbf{R}_{i}^{-1}\left(\mathbf{d}-\mathbf{m}_{i}\right)},
$$

for $i \in\{0,1\}$, where $\mathbf{m}_{0}=\left[\begin{array}{ll}0 & 0\end{array}\right]^{\mathrm{T}}$ and $\mathbf{m}_{1}=$ $\left[\sqrt{M} P_{\mathrm{rx}} \sqrt{M} P_{\mathrm{rx}}\right]^{\mathrm{T}}$. As a result, using the log-likelihood ratio (LLR), the hypothesis testing becomes

$$
T(\mathbf{d})=\left(\mathbf{d}-\mathbf{m}_{1}\right)^{\mathrm{H}} \mathbf{R}_{1}^{-1}\left(\mathbf{d}-\mathbf{m}_{1}\right)-\mathbf{d}^{\mathrm{H}} \mathbf{R}_{0}^{-1} \mathbf{d} \underset{H_{1}}{\stackrel{H_{0}}{<}} \lambda,
$$

where $\lambda>0$ is a decision threshold. While the LLR test can be carried out as in (26), it is not straightforward to find a decision boundary and error probabilities as $\mathbf{R}_{0} \neq \mathbf{R}_{1}$ [29]. However, thanks to the symmetric property of $\mathbf{R}_{1}$, the following result becomes useful to simplify the hypothesis testing.

Lemma 3: The test statistic in (26) can be expressed as

$$
T(\mathbf{d})=\frac{\left|y_{1}-u_{1}\right|^{2}}{\sigma_{1}^{2}}-\frac{\left|y_{1}\right|^{2}}{\rho_{1} \rho_{2}}+\left(\frac{1}{\sigma_{2}^{2}}-\frac{1}{\rho_{1} \rho_{2}}\right)\left|y_{2}\right|^{2},
$$

where $y_{1}=\sqrt{2} \Re(D), y_{2}=\sqrt{2} \Im(D), u_{1}=\sqrt{2 M} P_{\mathrm{rx}}, \sigma_{1}^{2}=$ $\rho_{1} \rho_{2}+P_{\mathrm{rx}}^{2}$, and $\sigma_{2}^{2}=\rho_{1} \rho_{2}-P_{\mathrm{rx}}^{2}$.

Proof: It can be shown that the eigenvalue decomposition of $\mathbf{R}_{1}$ becomes $\mathbf{R}_{1}=\mathbf{E} \operatorname{diag}\left(\sigma_{1}^{2}, \sigma_{2}^{2}\right) \mathbf{E}^{\mathrm{H}}$, where

$$
\mathbf{E}=\left[\mathbf{e}_{1} \mathbf{e}_{2}\right]=\frac{1}{\sqrt{2}}\left[\begin{array}{cc}
1 & 1 \\
1 & -1
\end{array}\right] .
$$

Here, $\mathbf{e}_{i}$ represents the eigenvector of $\mathbf{R}_{1}$ corresponding to the eigenvalue $\sigma_{i}^{2}$. Then, it is readily shown that $y_{i}=\mathbf{e}_{i}^{\mathrm{H}} \mathbf{d}$, 
$i=1,2$. In addition, $\mathbf{e}_{1}^{\mathrm{H}} \mathbf{m}_{1}=u_{1}$ and $\mathbf{e}_{2}^{\mathrm{H}} \mathbf{m}_{1}=0$. As a result, it can be shown that

$$
\begin{aligned}
& \left(\mathbf{d}-\mathbf{m}_{1}\right)^{\mathrm{H}} \mathbf{R}_{1}^{-1}\left(\mathbf{d}-\mathbf{m}_{1}\right) \\
& =\left[\left(y_{1}-u_{1}\right) y_{2}\right]^{*} \operatorname{diag}\left(\frac{1}{\sigma_{1}^{2}}, \frac{1}{\sigma_{2}^{2}}\right)\left[\left(y_{1}-u_{1}\right) y_{2}\right]^{\mathrm{T}},
\end{aligned}
$$

while

$$
\mathbf{d}^{\mathrm{H}} \mathbf{R}_{0}^{-1} \mathbf{d}=\frac{1}{\rho_{1} \rho_{2}} \mathbf{d}^{\mathrm{H}} \mathbf{E} \mathbf{E}^{\mathrm{H}} \mathbf{d}=\frac{1}{\rho_{1} \rho_{2}}\left(\left|y_{1}\right|^{2}+\left|y_{2}\right|^{2}\right) .
$$

Then, from (28) and (29), we have (27), which completes the proof.

From (27), it can be seen that $y_{1}$ plays a more significant role in the LLR test than $y_{2}$. Thus, the following simplified test statistic can be found:

$$
\bar{T}\left(y_{1}\right)=\frac{\left|y_{1}-u_{1}\right|^{2}}{\sigma_{1}^{2}}-\frac{\left|y_{1}\right|^{2}}{\rho_{1} \rho_{2}} .
$$

In fact, as shown below, $\bar{T}\left(y_{1}\right)$ is the LLR with $y_{1}$.

Lemma 4: Based on the CLT, under $H_{1}$ with $K_{q}=1$, we have

$$
y_{1}=\sqrt{2} \Re(D) \sim \mathcal{N}\left(u_{1}, \sigma_{1}^{2}\right) .
$$

In addition, $y_{1} \sim \mathcal{N}\left(0, \sigma_{0}^{2}\right)$ under $H_{0}$, where $\sigma_{0}^{2}=\rho_{1} \rho_{2}$.

Proof: Since $D$ is a CSCG random variable under $H_{0}$, it can be readily shown that the variance of $\sqrt{2} \Re(D)$ is $2 \frac{\operatorname{Var}(D)}{2}=\operatorname{Var}(D)=\rho_{1} \rho_{2}$. Under $H_{1}$, from (22) and (23), it can be shown that

$$
\begin{aligned}
\operatorname{Var}(D)= & \operatorname{Var}(\Re(D))+\operatorname{Var}(\Im(D))=\rho_{1} \rho_{2} \\
\mathbb{E}\left[(D-\mathbb{E}[D])^{2}\right]= & \operatorname{Var}(\Re(D))-\operatorname{Var}(\Im(D)) \\
& +j 2 \mathbb{E}[\Re(D-\mathbb{E}[D]) \Im(D-\mathbb{E}[D])]=P_{\mathrm{rx}}^{2},
\end{aligned}
$$

which implies that $\mathbb{E}[\Re(D-\mathbb{E}[D]) \Im(D-\mathbb{E}[D])]=0$, and $\operatorname{Var}(\Re(D))=\frac{\rho_{1} \rho_{2}+P_{\mathrm{rx}}^{2}}{2}$. From this, we can show that the variance of $y_{1}$ is $\sigma_{1}^{2}$. Furthermore, the mean of $y_{1}$ is $\sqrt{2} \mathbb{E}[\Re(D)]=\sqrt{2} \mathbb{E}[D]=u_{1}$. These complete the proof.

From Lemma 4, with the test statistic in (30), from [25], the LLR test can be simplified as follows:

$$
y_{1} \underset{H_{0}}{\stackrel{H_{1}}{<}} \tau \tau
$$

where $\tau$ is a decision threshold. Then, the probabilities of false alarm (FA) and missed detection (MD), denoted by $\mathbb{P}_{\mathrm{FA}}$ and $\mathbb{P}_{\mathrm{MD}}$, respectively, in the $\mathrm{S}$-preamble detection associated with (32) can be found as

$$
\begin{aligned}
\mathbb{P}_{\mathrm{FA}} & =\mathcal{Q}\left(\frac{\tau}{\sqrt{\sigma_{0}^{2}}}\right)=\mathcal{Q}\left(\frac{\tau}{\sqrt{\rho_{1} \rho_{2}}}\right) \\
\mathbb{P}_{\mathrm{MD}} & =\mathcal{Q}\left(\frac{u_{1}-\tau}{\sqrt{\sigma_{1}^{2}}}\right)=\mathcal{Q}\left(\frac{\sqrt{2 M} P_{\mathrm{rx}}-\tau}{\sqrt{\rho_{1} \rho_{2}+P_{\mathrm{rx}}^{2}}}\right) .
\end{aligned}
$$

For convenience, we can decide $\tau$ so that $\mathbb{P}_{\mathrm{FA}}=\mathbb{P}_{\mathrm{MD}}$. In this case, after some manipulations, we have

$$
\bar{\tau}=\frac{N_{0} \sqrt{2 M} \gamma}{1+\sqrt{1+\frac{\gamma^{2}}{\left(1+\kappa_{q_{1}} \gamma\right)\left(1+\kappa_{q_{2}} \gamma\right)}}},
$$

which leads to

$$
\mathbb{P}_{\mathrm{FA}}=\mathbb{P}_{\mathrm{MD}}=\mathcal{Q}\left(\sqrt{2 M} \theta\left(\gamma ; \kappa_{q_{1}}, \kappa_{q_{2}}\right)\right)
$$

where

$$
\theta\left(\gamma, \kappa_{q_{1}}, \kappa_{q_{2}}\right)=\frac{\gamma}{\kappa(\gamma)+\sqrt{\gamma^{2}+\kappa(\gamma)^{2}}}
$$

Here, $\kappa(\gamma)=\sqrt{\left(1+\kappa_{q_{1}} \gamma\right)\left(1+\kappa_{q_{1}} \gamma\right)}$. Since $\theta(\gamma)$ increases with $\gamma$ and it approaches $\frac{1}{\sqrt{\kappa_{q_{1}} \kappa_{q_{2}}}+\sqrt{1+\kappa_{q_{1}} \kappa_{q_{2}}}}$ as $\gamma \rightarrow \infty$, $\mathbb{P}_{\mathrm{FA}}=\mathbb{P}_{\mathrm{MD}}$ is lower-bounded by $\mathcal{Q}(\sqrt{2 M} c)$, where $c$ is a constant that is independent of $\gamma$ and $M$. This demonstrates that $M$ has to be large for a low probability of error.

Since $\left\{\kappa_{q_{1}}, \kappa_{q_{2}}\right\}$ are assumed to be known, there is no decision error when $\min \left\{\kappa_{q_{1}}, \kappa_{q_{2}}\right\}=0$ (i.e., in this case, without any decision error, S-preamble $q$ is decided as an unused one). Thus, the actual probability of FA can be lower than that in (33) or (35). Furthermore, we note that the error probability in (35) is a conditional probability as it depends on $\left\{\kappa_{q_{1}}, \kappa_{q_{2}}\right\}$. Thus, to find the average error probability, we need the distribution of $\left\{\kappa_{q_{1}}, \kappa_{q_{2}}\right\}$, which is difficult to derive as $\kappa_{q_{1}}$ and $\kappa_{q_{2}}$ are not independent. However, for tractable analysis, we can assume that they are independent. Under this assumption, for convenience, we use $\kappa$ that is the sum of randomly chosen $K$ elements of a row of $\boldsymbol{\Phi}$. As mentioned earlier, the number of 1's in a row of $\Phi$ is $L-1$. Thus, $\kappa$ can be approximated by a binomial random variable as follows:

$$
\kappa \sim \operatorname{Bin}\left(\bar{K}, \frac{L-1}{Q}\right), \bar{K} \leq \min \{K, L-1\} .
$$

Note that the maximum of $\kappa$ is $L-1$. Let $p_{k}=$ $\left(\begin{array}{c}\bar{K} \\ k\end{array}\right)\left(\frac{L-1}{Q}\right)^{k}\left(1-\frac{L-1}{Q}\right)^{\bar{K}-k}$ and

$$
[\mathbf{Q}]_{k, k^{\prime}}=\mathcal{Q}\left(\sqrt{2 M} \theta\left(\gamma ; k, k^{\prime}\right)\right), k, k^{\prime} \in\{0, \ldots, \bar{K}\} .
$$

Then, the average error probability can be approximately found as

$$
\mathbb{E}\left[\mathcal{Q}\left(\sqrt{2 M} \theta\left(\gamma ; \kappa_{q_{1}}, \kappa_{q, 2}\right)\right)\right] \approx \overline{\mathbb{P}}=\mathbf{p}^{\mathrm{T}} \mathbf{Q} \mathbf{p}
$$

where $\mathbf{p}=\left[\begin{array}{lll}p_{0} & \ldots & p_{\bar{K}}\end{array}\right]^{\mathrm{T}}$.

\section{Simulation Results}

In this section, for simulations, it is assumed that the channel vectors are generated according to Assumption A). We present simulation results with finite $M$ and $K$ and discuss their impact on the performance. For comparison, the conventional approach with (orthogonal) preambles is considered, while the approach in [16] is not discussed as its length of preamble transmission phase is a multiple of $L$, which results in a lower spectral efficiency ${ }^{1}$ than those of the proposed approach (i.e., $B=2$ ) and conventional approach (i.e., $B=1$ ).

We first consider the probabilities of MD and FA in detecting used or transmitted S-preambles in Section IV. The

\footnotetext{
${ }^{1}$ For a fair comparison with the approach in [16] in terms of throughput as well as spectral efficiency, the length of data packet has to be fixed. However, since the length of the data packet can be arbitrary, comparisons require more careful settings with details, which will be considered in the future as a further issue.
} 
decision threshold is set according to (34). Fig. 4 shows the error probabilities as functions of SNR, $\gamma$, when $M=100$, $L=32$, and $K=20$. In addition to the probabilities of MD and FA obtained from simulations, we show the average error probability in (39), which is an approximate average probability of error obtained by taking the average with respect to $\kappa_{q_{b}}$. As $\gamma$ increases, it is shown that the probabilities of FA and MD decrease as expected from (35) and (36). Furthermore, as mentioned earlier, it is observed that the probability of FA is lower than that of MD, while (39) is shown to be a reasonable approximate.

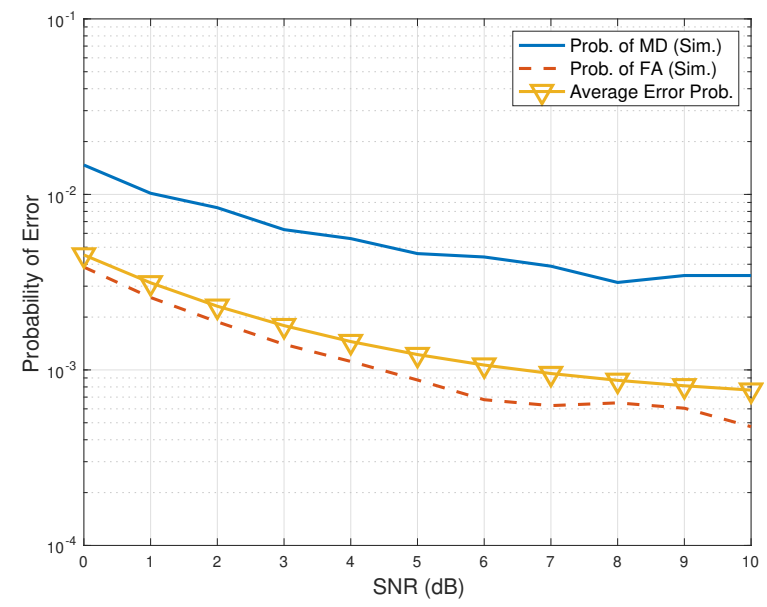

Fig. 4. Probabilities of MD and FA for S-preamble detection as functions of SNR, $\gamma$, when $M=100, L=32$, and $K=20$.

We present the probabilities of MD and FA for transmitted S-preamble detection as functions of $M$ when $K=20, L=$ 32 , and $\gamma=10 \mathrm{~dB}$ in Fig. 5. As $M$ increases, the probabilities of error decrease, which can be expected from (35) and (36).

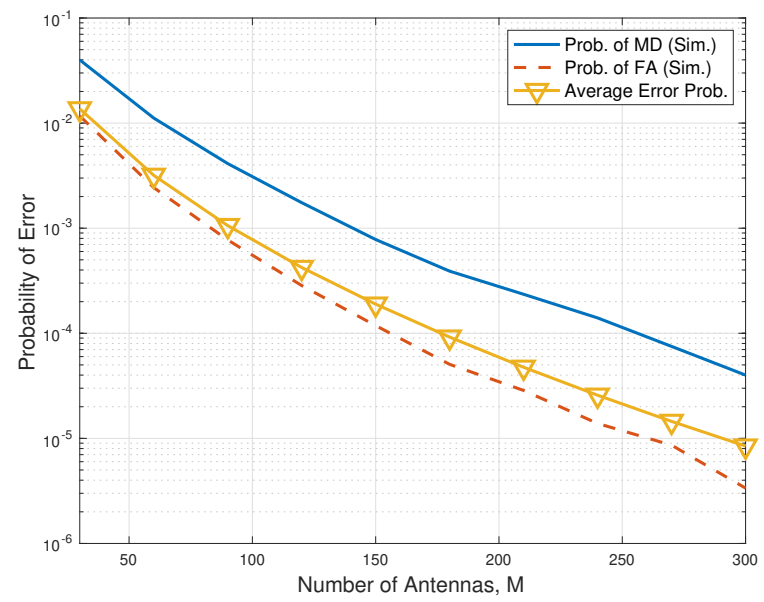

Fig. 5. Probabilities of MD and FA for S-preamble detection as functions of $M$ when $K=20, L=32$, and $\gamma=10 \mathrm{~dB}$.

Fig. 6 demonstrates the impact of $K$ on the error probabilities when $M=100, L=32$, and $\gamma=10 \mathrm{~dB}$. It is observed that as long as $K$ is less than $L$, the probabilities of MD and FA are sufficiently low. In other words, the detection of used Spreambles is successful for the channel estimation with a high probability if $K \leq L$, and the overall performance mainly depends on the following events: $i$ ) successful decoding (i.e., the SINR is higher than or equal to the threshold); ii) collision of S-preambles.

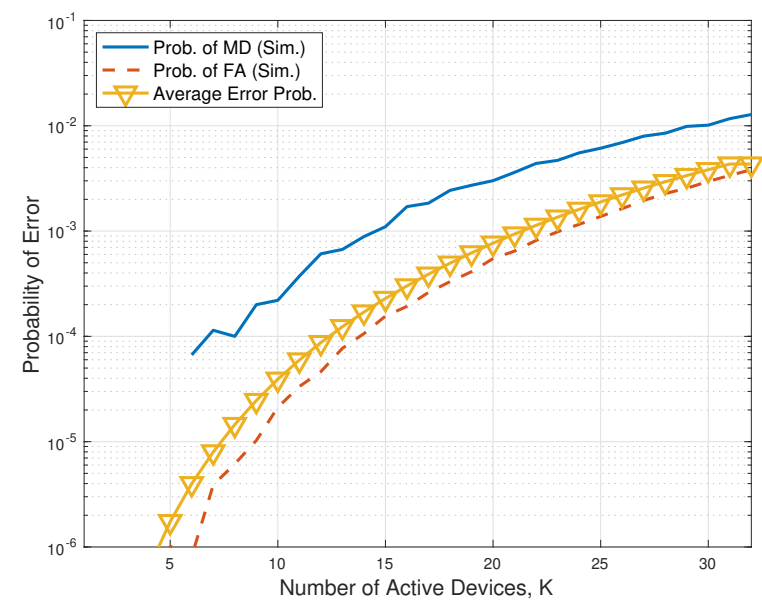

Fig. 6. Probabilities of MD and FA for S-preamble detection as functions of $K$ when $M=100, L=32$, and $\gamma=10 \mathrm{~dB}$.

We now present simulation results for the success probability, $\eta_{k}$. Conjugate beamforming is used with the estimated channel vectors in both approaches: the conventional approach with (orthogonal) preambles (i.e., $B=1$ ) and the proposed approach with $\mathrm{S}$-preambles (i.e., $B=2$ ). In the conventional approach, since each active device transmits one preamble, its transmit power becomes doubled for a fair comparison with the proposed one where each active device transmits a superposition of two different preambles, i.e., S-preamble. The theoretical success probability in (7) is shown with the asymptotic distribution of SINR obtained using the results in [12], which is shown by solid lines in Figs. $7-11$. Since this theoretical success probability is obtained without taking into consideration the rank deficiency shown in Fig. 3, it might be seen as an upper-bound.

In addition, to compare with the case of conventional nonorthogonal preambles, we consider $Q \mathrm{ZC}$ sequences of length $L$ and use the success probability derived in [17, Eq. 19], which is given by

$$
\mathbb{P}_{\text {succ }, \mathrm{ZC}}=\left(1-\frac{1}{Q}\right)^{K-1} \sum_{k=0}^{T}\left(\begin{array}{c}
K-1 \\
k
\end{array}\right) \alpha^{k}(1-\alpha)^{K-1-k}
$$

where $\alpha=1-\frac{L}{Q}$ and $T=\min \left\{\left\lfloor\frac{L}{\Omega}\right\rfloor, K-1\right\}$. Actually, (40) is an asymptotic probability with $M \rightarrow \infty$, in which case the noise is ignored, i.e., the SINR becomes the signal-interference ratio (SIR). As a result, (40) is independent of $M$ and $N_{0}$ (or SNR) and can be seen as an upper-bound.

Fig. 7 shows the success probability as a function of the target SINR, $\Omega$, when $M=100, K=16, L=32$, and $\gamma=10 \mathrm{~dB}$. In general, the success probability decreases with $\Omega$, while the success probability of the proposed approach with $\mathrm{S}$-preambles is higher than that of the conventional approach with preambles (especially, when $\Omega$ is sufficiently low, i.e., $\leq 6 \mathrm{~dB}$ ). As discussed earlier, the performance gain results 
from S-preambles that can effectively decrease the probability of preamble collision. It is also shown that the performance of non-orthogonal ZC preambles can be significantly degraded for $\Omega \geq 4 \mathrm{~dB}$ due to non-zero cross-correlation between nonorthogonal preambles. Note that although the length of $\mathrm{ZC}$ sequences has to be a prime, (40) is valid for any $L$. Thus, (40) is used for the results of ZC preambles in Figs. 7- 11.

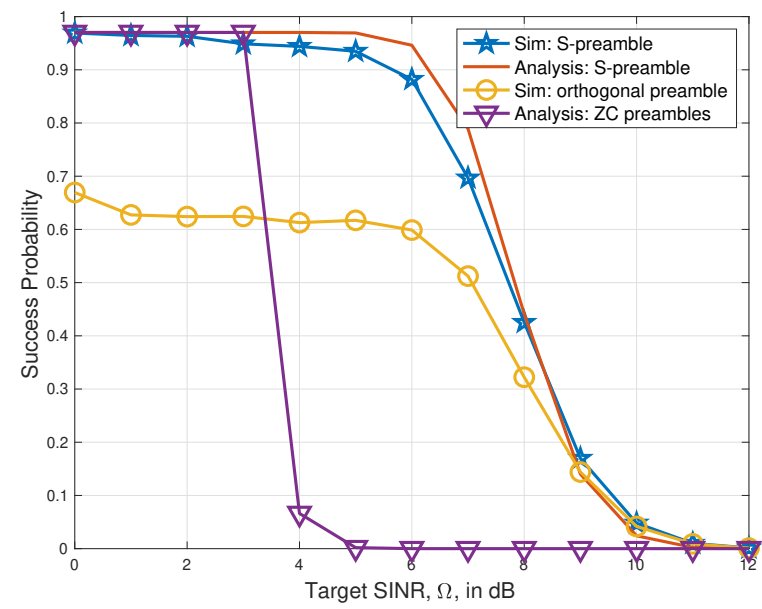

Fig. 7. Success probability as a function of the target SINR, $\Omega$, when $M=$ $100, K=16, L=32$, and $\gamma=10 \mathrm{~dB}$.

In Fig. 8, we show the success probability as a function of the SNR, $\gamma$, when $M=100, K=16, L=32$, and $\Omega=6 \mathrm{~dB}$. As the SNR increases, the success probability increases. It is noteworthy that the success probability of the proposed approach with S-preambles is lower than that of the conventional approach with preambles when $\gamma$ is low (e.g., $\leq 2 \mathrm{~dB}$ ). However, since a high success probability is desirable, it is expected to have a high SNR (e.g., $\geq 10$ $\mathrm{dB}$ ) in general, where the proposed approach outperforms the conventional approach. It is also shown that the performance of non-orthogonal ZC preambles is not satisfactory due to a high target SINR, $\Omega=6 \mathrm{~dB}$.

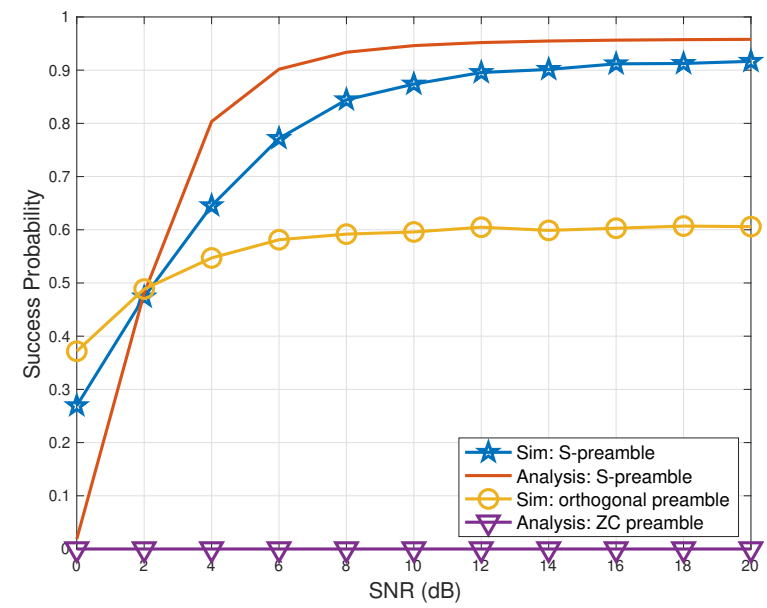

Fig. 8. Success probability as a function of the SNR, $\gamma$, when $M=100$, $K=16, L=32$, and $\Omega=6 \mathrm{~dB}$.

Fig. 9 shows the success probability as a function of the number of active devices, $K$, when $M=100, L=32, \gamma=$ $10 \mathrm{~dB}$, and $\Omega=6 \mathrm{~dB}$, where we can see that the success probability decreases with $K$. As expected, it is also shown that the proposed approach can support more active devices than the conventional approach. For example, with a success probability of 0.8 , the proposed approach can support around $K=17$ active devices, while the conventional approach can support around $K=8$. The performance of non-orthogonal $\mathrm{ZC}$ preambles is degraded quickly as $K$ increases and becomes poor when $K \geq 10$.

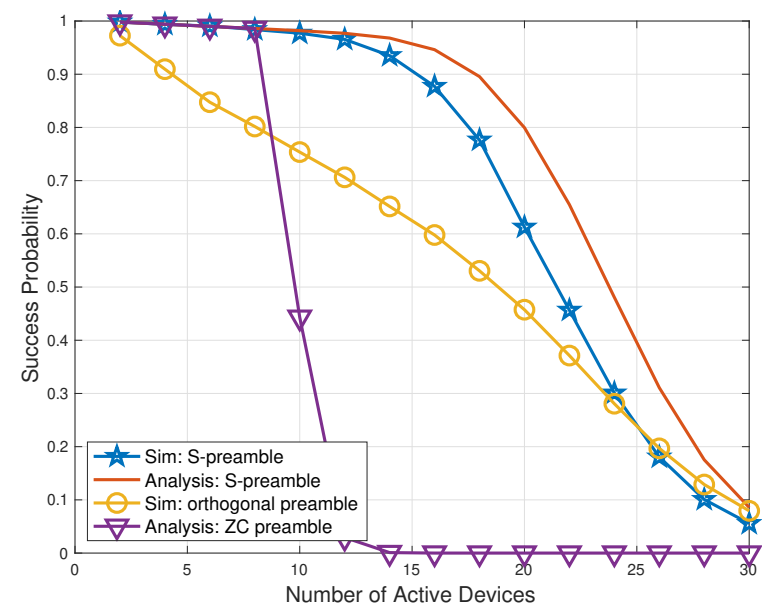

Fig. 9. Success probability as a function of the number of active devices, $K$, when $M=100, L=32, \gamma=10 \mathrm{~dB}$, and $\Omega=6 \mathrm{~dB}$.

In Fig. 10, the success probability is shown as a function of the number of preambles, $L$, when $K=20, \Omega=6 \mathrm{~dB}$, and $(M, \gamma)=\{(100,10 \mathrm{~dB}),(500,30 \mathrm{~dB})\}$. As mentioned earlier, the theoretical success probability does not take into account the rank deficiency of $\overline{\boldsymbol{\Phi}}$ in Fig. 3, which is more serious when $L$ is close to $K$. Thus, the theoretical success probability becomes not tight when $L$ is small. However, it becomes tight as $L$ increases as shown in Fig. 10. Note that the performance of non-orthogonal ZC preambles from (40) is an asymptotic result when $M \rightarrow \infty$. Thus, as $L \rightarrow \infty$ with a fixed $K$, the asymptotic success probability is only dependent on preamble collision and becomes $\left(1-\frac{1}{Q}\right)^{K-1} \rightarrow 1$ as shown in Fig. 10 . Thus, (40) has to be regarded as an upper-bound and can be used to compare with the proposed approach when $M$ is sufficiently large, i.e., the results in Fig. 10 (b). In Fig. 10 (b), it is clearly shown that $L$ has to be sufficiently large for a low cross-correlation when ZC sequences are used so that the SIR is higher than or equal to the threshold SINR. Otherwise, the success probability is low.

Finally, Fig. 11 shows the success probability as a function of the number of antennas, $M$, when $K=16, L=32, \gamma=10$ $\mathrm{dB}$, and $\Omega=6 \mathrm{~dB}$. It is shown that the success probability increases with $M$ in both the proposed and conventional approaches. However, although $M \rightarrow \infty$, it is shown that the success probability does not approach 1 in Fig. 11 due to preamble collision as discussed earlier. In other words, while massive MIMO can provide a high SINR, its performance is limited by preamble collision. Since the proposed approach 


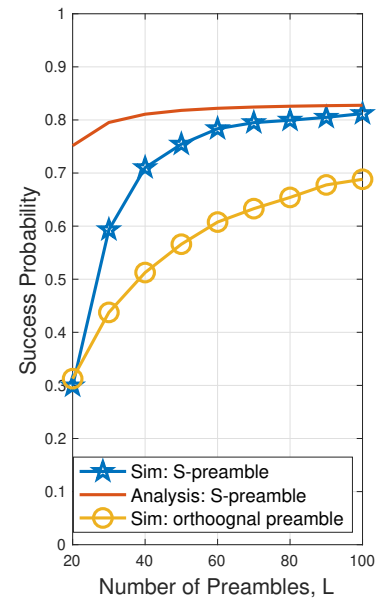

(a)

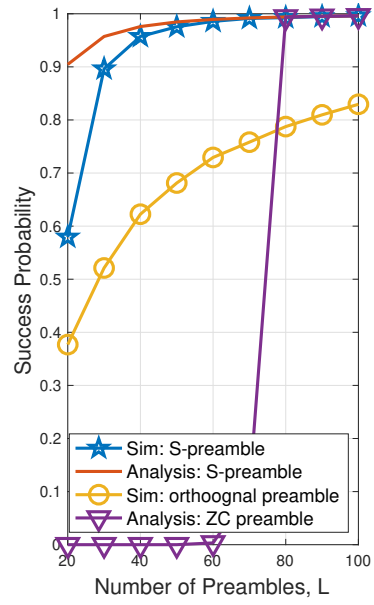

(b)
Fig. 10. Success probability as a function of the number of preambles, $L$, when $K=20$ and $\Omega=6 \mathrm{~dB}$ : (a) $M=100$ and $\gamma=10 \mathrm{~dB}$; (b) $M=500$ and $\gamma=30 \mathrm{~dB}$.

with S-preambles can effectively reduces the probability of preamble collision, it can improve the performance and has a higher success probability than the conventional approach. The success probability in (40) for the performance of ZC preambles is almost 0 as the SINR is lower than $\Omega=6 \mathrm{~dB}$ due to non-zero cross-correlation between $\mathrm{ZC}$ preambles.

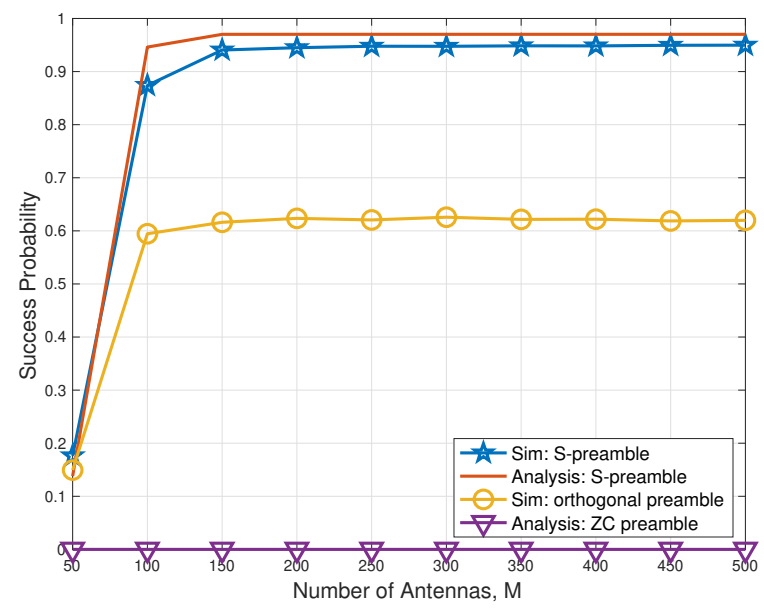

Fig. 11. Success probability as a function of the number of antennas, $M$, when $K=16, L=32, \gamma=10 \mathrm{~dB}$, and $\Omega=6 \mathrm{~dB}$.

\section{CONCLUding REMARKS}

In this paper, we proposed the use of S-preambles for a grant-free random access scheme with massive MIMO to improve the performance (e.g., to support more active devices). Without increasing the size of preamble pool and the length of preamble transmission phase, it was shown that the proposed approach can effectively reduce preamble collisions using Spreambles and increase the success probability.

Since S-preambles can be seen as structured non-orthogonal preambles, we believe that there would be different approaches to design non-orthogonal preambles with effective channel estimation methods, which might be an interesting topic to be studied in the future.

\section{REFERENCES}

[1] C. Bockelmann, N. Pratas, H. Nikopour, K. Au, T. Svensson, C. Stefanovic, P. Popovski, and A. Dekorsy, "Massive machine-type communications in 5G: physical and MAC-layer solutions," IEEE Communications Magazine, vol. 54, pp. 59-65, Sep 2016.

[2] Z. Dawy, W. Saad, A. Ghosh, J. G. Andrews, and E. Yaacoub, "Toward massive machine type cellular communications," IEEE Wireless Communications, vol. 24, pp. 120-128, Feb 2017.

[3] 3GPP TR $37.868 \mathrm{~V} 11.0$, Study on RAN improvments for machine-type communications, October 2011.

[4] 3GPP TS 36.321 V13.2.0, Evolved Universal Terrestrial Radio Access (E-UTRA); Medium Access Control (MAC) protocol specification, June 2016.

[5] C. H. Chang and R. Y. Chang, "Design and analysis of multichannel slotted ALOHA for machine-to-machine communication," in Proc. IEEE GLOBECOM, pp. 1-6, Dec 2015.

[6] J. Choi, "On the adaptive determination of the number of preambles in RACH for MTC," IEEE Communications Letters, vol. 20, pp. 13851388, July 2016.

[7] T. L. Marzetta, "Noncooperative cellular wireless with unlimited numbers of base station antennas," IEEE Trans. Wireless Communications, vol. 9, pp. 3590-3600, Nov. 2010.

[8] E. Björnson, J. Hoydis, and L. Sanguinetti, "Massive MIMO has unlimited capacity," IEEE Trans. Wireless Communications, vol. 17, pp. 574-590, Jan 2018.

[9] E. de Carvalho, E. Björnson, J. H. Sørensen, E. G. Larsson, and P. Popovski, "Random pilot and data access in massive MIMO for machine-type communications," IEEE Trans. Wireless Communications, vol. 16, pp. 7703-7717, Dec 2017.

[10] K. Senel and E. G. Larsson, "Device activity and embedded information bit detection using AMP in massive MIMO," in 2017 IEEE Globecom Workshops (GC Wkshps), pp. 1-6, Dec 2017.

[11] L. Liu, E. G. Larsson, W. Yu, P. Popovski, C. Stefanovic, and E. de Carvalho, "Sparse signal processing for grant-free massive connectivity: A future paradigm for random access protocols in the Internet of Things," IEEE Signal Processing Magazine, vol. 35, pp. 88-99, Sept 2018.

[12] J. Ding, D. Qu, H. Jiang, and T. Jiang, "Success probability of grant-free random access with massive MIMO," IEEE Internet of Things J., vol. 6, pp. 506-516, Feb 2019

[13] C. Bockelmann, N. K. Pratas, G. Wunder, S. Saur, M. Navarro, D. Gregoratti, G. Vivier, E. De Carvalho, Y. Ji, C. Stefanović, P. Popovski, Q. Wang, M. Schellmann, E. Kosmatos, P. Demestichas, M. RacealaMotoc, P. Jung, S. Stanczak, and A. Dekorsy, "Towards massive connectivity support for scalable mMTC communications in $5 \mathrm{G}$ networks," IEEE Access, vol. 6, pp. 28969-28992, 2018.

[14] J. Choi, "Two-stage multiple access for many devices of unique identifications over frequency-selective fading channels," IEEE Internet of Things J., vol. 4, pp. 162-171, Feb 2017.

[15] J. Choi, "On throughput of compressive random access for one short message delivery in IoT," IEEE Internet of Things J., vol. 7, no. 4, pp. 3499-3508, 2020.

[16] H. Jiang, D. Qu, J. Ding, and T. Jiang, "Multiple preambles for high success rate of grant-free random access with massive MIMO," IEEE Trans. Wireless Communications, vol. 18, pp. 4779-4789, Oct 2019.

[17] J. Ding, D. Qu, and J. Choi, "Analysis of non-orthogonal sequences for grant-free RA with massive MIMO," IEEE Trans. Communications, vol. 68, pp. 150-160, Jan 2020.

[18] D. Donoho, "Compressed sensing," IEEE Trans. Information Theory, vol. 52, pp. 1289-1306, April 2006

[19] E. Candes, J. Romberg, and T. Tao, "Robust uncertainty principles: exact signal reconstruction from highly incomplete frequency information," IEEE Trans. Information Theory, vol. 52, pp. 489-509, Feb 2006.

[20] H. Seo, J. Hong, and W. Choi, "Low latency random access for sporadic MTC devices in internet of things," IEEE Internet of Things J., vol. 6 , pp. 5108-5118, June 2019.

[21] J. Choi, "NOMA-based compressive random access using Gaussian spreading," IEEE Trans. Communications, vol. 67, no. 7, pp. 5167-5177, 2019.

[22] E. Björnson, E. G. Larsson, and M. Debbah, "Massive MIMO for maximal spectral efficiency: How many users and pilots should be allocated?," IEEE Trans. Wireless Communications, vol. 15, pp. 12931308, Feb 2016. 
[23] A. S. Asratian, T. M. J. Denley, and R. Häggkvist, Bipartite Graphs and Their Applications. New York, NY, USA: Cambridge University Press, 1998.

[24] L. Scharf and C. Demeure, Statistical Signal Processing: Detection, Estimation, and Time Series Analysis. Addison-Wesley series in electrical and computer engineering, Addison-Wesley Publishing Company, 1991.

[25] S. Kay, Fundamentals of Statistical Signal Processing: Detection theory. Prentice Hall Signal Processing Series, Prentice-Hall PTR, 1998.

[26] R. K. Mallik and N. C. Sagias, "Distribution of inner product of complex gaussian random vectors and its applications," IEEE Trans. Communications, vol. 59, pp. 3353-3362, December 2011.

[27] B. Picinbono, "Second-order complex random vectors and normal distributions," IEEE Trans. Signal Processing, vol. 44, pp. 2637-2640, Oct 1996.

[28] A. van den Bos, "The multivariate complex normal distribution-a generalization," IEEE Trans. Information Theory, vol. 41, pp. 537-539, March 1995.

[29] R. O. Duda, P. E. Hart, and D. G. Stork, Pattern Classification (2nd Edition). New York, NY, USA: Wiley-Interscience, 2000. 